\title{
Correlation of the Levels of Vitamin D with Size of Leiomyoma in a 450-bedded Maternity Hospital of Delhi
}

\author{
Vinita Sarbhai ${ }^{1}$, Sangita N Ajmani ${ }^{2}$, Shobhika Singh ${ }^{3}$
}

\begin{abstract}
Introduction: The pathogenesis of fibroids has been hypothesized to involve a positive feedback loop between extracellular matrix production and cell proliferation, and vitamin D might act to block the positive feedback. Hence, this study was carried out to know the correlation of vitamin D levels with the size of fibroids.

Methods: Seventy-five patients diagnosed with leiomyoma on ultrasound scan were labeled as the case group. Control group comprised of 75 women whose pelvic ultrasound was normal and there was no fibroid. On trans-vaginal sonography (TVS), we measured the volume of each of them. The quantitative detection of serum vitamin D levels was performed using a kit based on chemiluminescence in cases and control.

Results: The mean value of vitamin D levels in cases was $12.58+4.09 \mathrm{ng} / \mathrm{mL}$ while in controls it was $18.99+5.72 \mathrm{ng} / \mathrm{mL}$ with $p$-value $<0.001$. Thus it was statistically significantly lower in cases than in controls. Pearson correlation of total volume of the fibroid $\left(\mathrm{cm}^{3}\right)$ and vitamin D level ( $\mathrm{ng} / \mathrm{mL}$ ) was -0.292 with $p$-value of 0.011 .

Conclusion: The volume of fibroid and serum vitamin D levels are inversely related to each other, i.e., the less is the vitamin D levels, the more will be the volume of the fibroid.

Keywords: Fibroid uterus, Leiomyoma uteri, Vitamin D.

Journal of South Asian Federation of Obstetrics and Gynaecology (2021): 10.5005/jp-journals-10006-1869
\end{abstract}

\section{INTRODUCTION}

Uterine fibroids or leiomyoma is the most common benign tumors of the female genital system. Leiomyoma is the localized proliferation of smooth muscles of the uterus, surrounded by a psuedocapsule. The most common presentation of uterine fibroids is during the middle and late reproductive years, in the form of menorrhagia, chronic pelvic pain, and pressure symptoms including bowel and bladder symptoms. It also affects fertility prospects by causing infertility, recurrent pregnancy loss, and other pregnancy complications. It is also one of the leading causes of hysterectomy and gynecologic hospitalizations, leading to a tremendous economic burden.

Vitamin D has been shown to result in reduction in cellular proliferation, increase in apoptosis, promote differentiation, and control numerous other processes at basic tissue levels like vascular angiogenesis, extracellular matrix synthesis, and other immune mechanisms, in various studies. ${ }^{1}$ It has been hypothesized that myoproliferation in fibroids might result due to a positive feedback cycle of extracellular matrix production and cell proliferation ${ }^{2}$ and vitamin D helps to block this feedback and prevent the cellular proliferation which results in fibroid generation.

Vitamin $D$ downregulates the expression of the genes $P C N A, C D K 1$, and $B C L-2$ in cultured human leiomyoma cells and inhibits the action of catechol-O-methyltransferase in them with the resultant inhibition of their growth. ${ }^{3-5}$ The calcitriol or $1,25(\mathrm{OH})_{2} \mathrm{D}_{3}$ ), which is the active form of vitamin $\mathrm{D}$, works at cellular level by activating cellular signaling pathways like the classic nuclear and rapid nongenomic pathways. In the classic nuclear pathway, $1,25(\mathrm{OH})_{2} \mathrm{D}_{3}$ forms a complex with receptors like VDR and $R X R$ that binds the vitamin $D$ response elements to modulate the expression of target genes. In the rapid nongenomic pathway,
${ }^{1-3}$ Department of Gynaecology and Obstetrics, Kasturba Hospital, Delhi, India

Corresponding Author: Vinita Sarbhai, Department of Gynaecology and Obstetrics, Kasturba Gandhi Hospital, Delhi, India, e-mail: vinitasarbhai@gmail.com

How to cite this article: Sarbhai V, Ajmani SN, Singh S. Correlation of the Levels of Vitamin D with Size of Leiomyoma in a 450-bedded Maternity Hospital of Delhi. J South Asian Feder Obst Gynae 2021;13(2):77-80.

Source of support: Nil

Conflict of interest: None

$1,25(\mathrm{OH})_{2} \mathrm{D}_{3}$ binds to membrane-bound vitamin $\mathrm{D}$ receptors, resulting in the activation of various signaling pathways. ${ }^{6}$ These pathways then modulate several processes including G0/G1 progression, apoptosis, differentiation, and angiogenesis. ${ }^{6}$

When calcitriol is added to fibroid tissue, in laboratory culture, it leads to suppression of cellular proliferation and extracellular matrix synthesis. Vitamin $D$ is also a potent antitumor agent that effectively suppresses the proliferation of human uterine leiomyoma cells in vitro and has been shown to reduce fibroid volume in laboratory studies on animals ${ }^{7-9}$; hence, this study of levels of serum vitamin D in women having leiomyoma is planned.

\section{Materials and Methods}

The study was conducted in the department of Obstetrics and Gynecology at Kasturba Hospital, a 450-bedded tertiary care hospital, which is located in the walled city of Delhi. The ethical clearance was taken from the institutional ethics committee. The 
study subjects were recruited, from January to December 2017, after a written informed consent, from patients attending the clinic of Kasturba Hospital.

Case group: Comprised 75 women who were diagnosed with leiomyoma with a mean diameter more than $10 \mathrm{~mm}$ at an ultrasound.

Control group: Comprised 75 women whose pelvic ultrasound was normal and there was no fibroid.

\section{Inclusion Criteria}

- Women of age-group 18 to 50 years diagnosed with leiomyoma on ultrasound scan.

\section{Exclusion Criteria}

- Pregnant women, menopausal women, women on hormonal treatment (e.g., oral contraceptive pills) during the past 3 months.

- Vitamin D supplementation in last 6 months.

- Patients currently lactating or lactating in last 6 months.

- Patients having a history of prior myomectomy.

- Women diagnosed with adenomyosis and other causes of abnormal uterine bleeding.

- Patients reporting chronic medical problems were excluded from both study groups.

- Patients having malignancy, multiple sclerosis, autoimmune disorders

- Women having leiomyoma less than $10 \mathrm{~mm}$ on TVS

A detailed history of clothing, outdoor activity, fish intake was taken, followed by basic investigations, like hemogram, blood sugar LFT, KFT, and urine routine microscopy. Perpendicular diameters were measured for each myoma in three directions, and the volume of each of them was recorded on TVS. Then the total fibroid volume was recorded by adding all the individual fibroids volume. Venous blood sample was collected from selected women for the quantitative estimation of serum vitamin D levels. The estimation of serum vitamin D levels was performed using a commercially available kit of chemiluminescence assay. Women were grouped into three different groups, according to World Health Organization recommendations.

- Vitamin D deficient-if the levels were $<10 \mathrm{ng} / \mathrm{mL}$

- Vitamin D insufficient - with levels between 10 and $19.9 \mathrm{ng} / \mathrm{mL}$

- Vitamin D sufficient—vitamin D levels were $>20 \mathrm{ng} / \mathrm{mL}$

Pelvic USG: The following parameters were evaluated:

- Diameters were taken in three perpendicular directions, and total uterine size was calculated using the formula for estimation of the volume of the elongated ellipse $(x \times y \times z \times 0.523)$, where $x$ is height, $y$ is width, and $z$ is depth.

- Volume of all fibroids was calculated using the formula for estimation of volume of prolate ellipse $(x \times y \times z \times 0.523)$, where $x$ is height, $y$ is width, and $z$ is depth.

- Fibroids were classified according to the International Federation of Gynaecology and Obstetrics uterine leiomyoma classification with respect to position and location of each fibroid, like subserosal, intramural, or submucosal.

- Any other features, like echogenicity, calcifications, necrosis, and degeneration, etc., of each fibroid lesion were recorded.

- Other adnexal findings like ovary were noted.
The data so obtained were entered in the statistical package for the social science system version SPSS 17.0 and statistical evaluation was performed. If $p$-value was $<0.05$, it was considered statistically significant.

\section{Results}

The values of serum vitamin D were estimated in 75 cases of leiomyoma and 75 controls. The mean level of vitamin D in cases was $12.58 \pm 4.09 \mathrm{ng} / \mathrm{mL}$ while in controls it was $18.99 \pm 5.72 \mathrm{ng} /$ $\mathrm{mL}$ with $p$-value of 0.001 . The levels of vitamin D were significantly less in cases than in controls. Thus, it was statistically significant in cases and controls (Table 1).

\section{Volume of Fibroids with Levels of Vitamin D}

Out of our 75 cases, 32 were in vitamin D deficient category (42.7\%) while 43 were in vitamin D insufficient category (57.3\%) and only six cases had vitamin D in normal range (8\%) (Table 2). The mean volume of fibroid in cases was $39.12 \pm 90.78 \mathrm{~cm}^{3}$ (Table 3). The fibroid with larger volume was more vitamin $D$ deficient as compared to fibroid with lower volume with $p$-value of 0.044 (Table 2; Fig. 1).

Then a graph between correlations in terms of the strength and direction of the relationship between the total volume of fibroid $\left(\mathrm{cm}^{3}\right)$ and vitamin D level $(\mathrm{ng} / \mathrm{mL})$ was plotted. Pearson correlation of total volume of fibroid $\left(\mathrm{cm}^{3}\right)$ and vitamin D level $(\mathrm{ng} / \mathrm{mL})$ was -0.292 with $p$-value of 0.011 (Graph 1; Table 4).

The mean volume of fibroid in cases was $39.12 \pm 90.78 \mathrm{~cm}^{3}$. Then, we plotted a graph for cases between volume of fibroid and levels of vitamin D.

Graph 1 and Table 4 show correlations in terms of the strength and direction of the relationship between total volume of fibroid $\left(\mathrm{cm}^{3}\right)$ and vitamin D level $(\mathrm{ng} / \mathrm{mL})$. Pearson correlation of total volume of fibroid and vitamin $D$ level $(\mathrm{ng} / \mathrm{mL})=-0.292$ with $p$-value $\leq 0.011$.

In conclusion, it indicates that the strength of association between the variables is moderate and negative in nature $(r=-0.292)$ and that the correlation coefficient is significant $(p<0.011)$. This indicates that lower the levels of vitamin D higher is the size of fibroid.

\section{Discussion}

The mean serum vitamin $D$ levels of cases were $12.58 \pm 4.09 \mathrm{ng} /$ $\mathrm{mL}$ while in controls it was $18.99 \pm 5.72 \mathrm{ng} / \mathrm{mL}$ with $p$-value $<0.001$. Thus, it was statistically significantly lower in the cases than in the controls.

Vitamin D levels were divided according to WHO, i.e., vitamin $D$ deficient (level $<10 \mathrm{ng} / \mathrm{mL}$ ), vitamin $D$ insufficient (level between 10 and $20 \mathrm{ng} / \mathrm{mL}$ ), and vitamin $D$ sufficient (level $>20 \mathrm{ng} / \mathrm{mL}$ ). It was observed that $42.7 \%$ of our cases were vitamin D deficient, while $49.9 \%$ of cases were vitamin D insufficient. However $52 \%$ of our controls were vitamin D sufficient, $28 \%$ of controls were vitamin D insufficient, and only $20 \%$ of controls were vitamin D deficient.

Table 1: Mean levels of serum vitamin $D$ in case and control groups

\begin{tabular}{llll}
\hline \multirow{2}{*}{ Mean vitamin $D(\mathrm{ng} / \mathrm{mL})$} & Cases & Controls & \\
\cline { 2 - 4 } & Mean $\pm S D$ & Mean $\pm S D$ & p value \\
Vitamin D level $(\mathrm{ng} / \mathrm{mL})$ & $12.58 \pm 4.09$ & $18.99 \pm 5.72$ & $<0.001$ \\
\hline
\end{tabular}


Table 2: Correlation of volume of fibroids with levels of vitamin $D$ in case group

\begin{tabular}{|c|c|c|c|c|c|c|c|c|}
\hline \multirow{2}{*}{ Total volume of fibroid, $\mathrm{cm}^{3}$} & \multirow{2}{*}{ Total cases } & \multicolumn{2}{|c|}{ Vitamin D level $<10$} & \multicolumn{2}{|c|}{ Vitamin D level 10-20 } & \multicolumn{2}{|c|}{ Vitamin D Level > 20} & \multirow{2}{*}{$p$ value } \\
\hline & & Frequency & $\%$ & Frequency & $\%$ & Frequency & $\%$ & \\
\hline $0-5$ & 9 & 1 & 11.1 & 6 & 66.7 & 2 & 22.2 & \\
\hline $5.1-10$ & 22 & 6 & 27.3 & 15 & 68.2 & 1 & 4.5 & \\
\hline $10.1-15$ & 9 & 2 & 22.2 & 6 & 66.7 & 1 & 11.1 & \\
\hline $15.1-20$ & 8 & 5 & 62.5 & 2 & 25.0 & 0 & 0.0 & \\
\hline $20.1-30$ & 7 & 4 & 57.1 & 2 & 28.6 & 2 & 28.6 & 0.044 \\
\hline $30.1-50$ & 9 & 8 & 88.9 & 5 & 55.6 & 0 & 0.0 & \\
\hline $50.1-100$ & 6 & 2 & 33.3 & 0 & 0.0 & 0 & 0.0 & \\
\hline$>100$ & 5 & 4 & 80.0 & 1 & 20.0 & 0 & 0.0 & \\
\hline Total & 75 & 32 & 42.7 & 43 & 57.3 & 6 & 8.0 & \\
\hline
\end{tabular}

Table 3: Mean of volume of fibroids in case group

\begin{tabular}{llll}
\hline & \multicolumn{3}{c}{ Cases } \\
\cline { 2 - 4 } & Mean $\pm S D$ & Median & Min-max \\
\hline Total volume of fibroid & $39.12 \pm 90.78$ & 12.14 & $2.1-702.9$ \\
$(a \times b \times c \times 0.523)$, where $a$ & & & \\
is height, $b$ is width and $c$ is & & \\
length $\left(\mathrm{cm}^{3}\right)$ & & \\
\hline
\end{tabular}

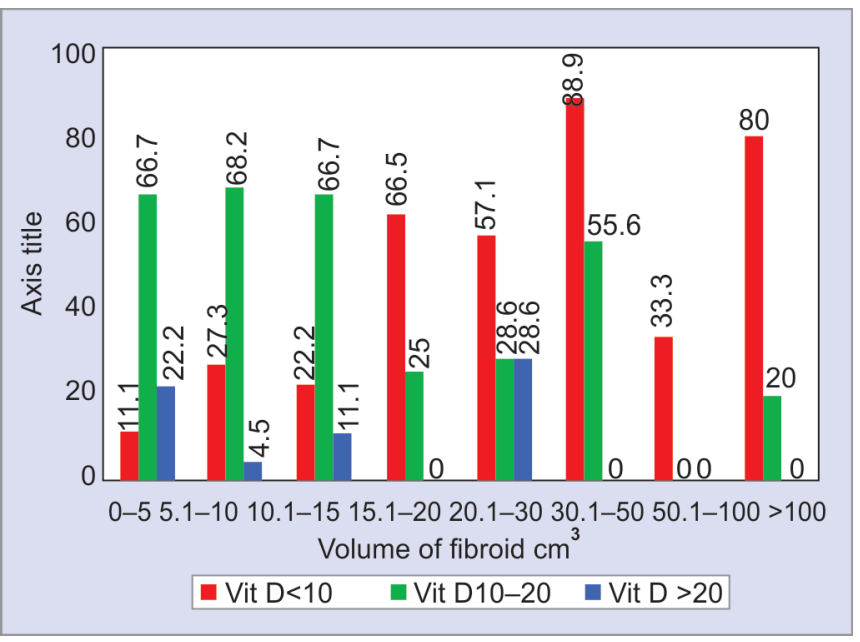

Fig. 1: Correlation between the total volume of fibroid and vitamin D in cases

The mean volume of fibroid of our cases was $39.12 \pm 90.78 \mathrm{~cm}^{3}$. Then, we plotted a graph between correlations in terms of the strength and direction of the relationship between total volume of fibroid $\left(\mathrm{cm}^{3}\right)$ and vitamin D level $(\mathrm{ng} / \mathrm{mL})$. Pearson correlation of total volume of fibroid $\left(\mathrm{cm}^{3}\right)$ and vitamin $D$ level $(\mathrm{ng} / \mathrm{mL})$ was -0.292 with $p$-value of 0.011 .

In conclusion, it indicates that the strength of association between the variables is moderate and negative in nature $(r=-0.292)$ and that the correlation coefficient is significant $(p<0.011)$. This means that volume of fibroid and serum vitamin D levels are inversely related to each other, i.e., the less is the vitamin D levels more will be the volume of fibroid.

Sabry in 2013 also documented a substantial inverse correlation between total fibroid volume and vitamin D levels $(r=-0.31$;

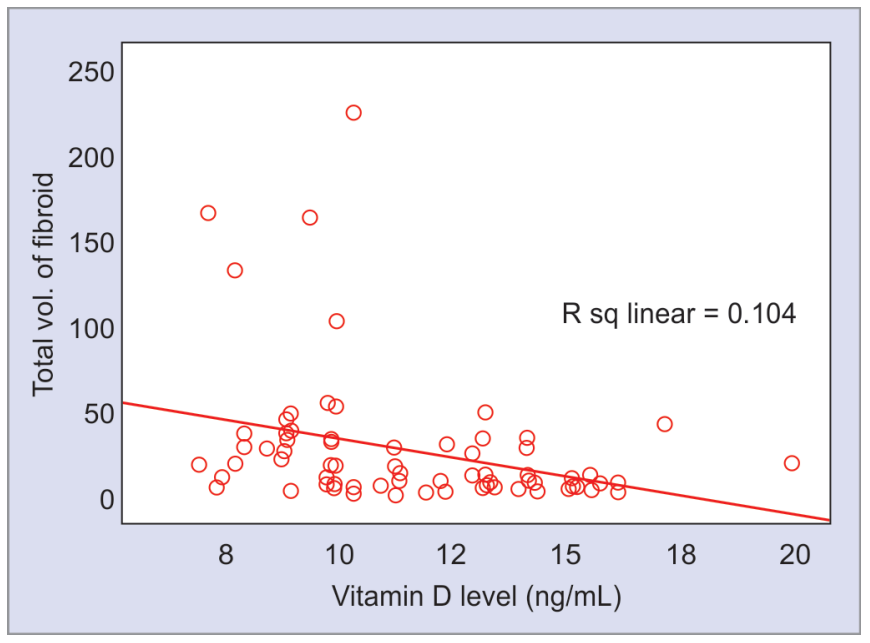

Graph 1: Volume of fibroids in cases vs levels of Vitamin D in cases

Table 4: Correlation coefficient

\begin{tabular}{llc}
\hline Total volume of fibroid & Correlation coefficient & -0.292 \\
$(a \times b \times c \times 0.523)$, where $a$ & $p$ value & 0.011 \\
is height, $b$ is width, and $c$ is & $N$ & 75 \\
length $\left(\mathrm{cm}^{3}\right)$ & & \\
\hline
\end{tabular}

$p=0.002$ ) in women with fibroid uterus. Patients with larger fibroid volumes were associated with lower serum vitamin D levels and vice versa. ${ }^{10}$

Ciavattini in 2016 documented that the baseline serum vitamin $D$ levels were inversely proportional to the volume of the largest fibroid $(r=-0.18, p=0.01)$ and the total volume of fibroids $(r=-0.19, p=0.01)$ in his study group of 108 women diagnosed with leiomyoma. ${ }^{11}$

Thus, there is an inverse relationship between serum vitamin D levels and the volume of fibroid. In the present study also, there is a negative correlation between serum vitamin D levels and leiomyoma size in individuals having fibroids, which was found to be statistically significant. The largest fibroid tumor volume was associated with the lowest serum vitamin D levels. This definitely establishes a direct relationship and dose-effect pattern with respect to levels of vitamin D and fibroid volume and gives strong evidence for its role in the etiopathogenesis of leiomyoma. 


\section{Conclusions and Recommendations}

Lower serum vitamin D levels were inversely correlated with leiomyoma burden. The mean volume of fibroid of our cases was $39.12 \pm 90.78 \mathrm{~cm}^{3}$. Pearson correlation of total volume of fibroid and vitamin D level $(\mathrm{ng} / \mathrm{mL})$ was -0.292 with $p$-value of 0.011 . In conclusion, it indicates that the strength of association between the variables is moderate and negative in nature $(r=-0.292)$ and that the correlation coefficient is significant $(p<0.011)$. This means that volume of fibroid and serum vitamin $D$ levels are inversely related to each other, i.e., the less is the vitamin D levels more will be the volume of fibroid. This study concluded that lower levels of vitamin D were related to bigger leiomyoma size, in terms of total volume of fibroids.

Supplementation of vitamin $D$ is a potential regulator of development of uterine leiomyoma. These data are of particular clinical relevance, since vitamin D could be a potentially safe, nonsurgical modality for the treatment and prevention of uterine fibroids. Appropriate and well-designed randomized controlled trials are certainly required, in order to confirm the utility and efficacy of oral supplementation of vitamin D in preventing, inhibiting, or stabilizing the growth of uterine fibroids and to determine the appropriate dose requirement and duration of its treatment.

The current methods available for management are surgical methods like myomectomy, hysterectomy, embolization, or medical treatment, such as gonadotropin-releasing hormone analog, or selective progesterone receptor modulators. They are relatively effective in the treatment and prevention of the symptoms but are expensive and are not devoid of side effects. Thus, it is essential to identify high-risk patients who are prone to develop leiomyoma and implement fibroid prevention in them. Hence, it is pertinent to conduct randomized clinical trials and determine the efficacy of vitamin D supplementation for treatment/ prevention of uterine fibroids. If there are positive results, vitamin $D$ could become a new modality in the management of fibroid uterus, as it is easily available, inexpensive, safe, and with supplementary pleiotropic effects in the prevention and treatment of leiomyoma.

Ethics approval and consent to participate: This study was approved by the institutional ethical committee and informed consent was taken from all participants.

\section{References}

1. Holick MF. Vitamin D deficiency. N Engl J Med 2007;357(3):266-281. DOI: 10.1056/NEJMra070553.

2. Leppert PC, Catherino WH, Segars JH. A new hypothesis about the origin of uterine fibroids based on gene expression profiling with microarrays. Am J Obstet Gynecol 2006;195(2):415-420. DOI: 10.1016/j.ajog.2005.12.059.

3. Sabry M, Al-Hendy A. Innovative oral treatments of uterine leiomyoma. Obstet Gynecol Int 2012;2012:943-963. DOI: $10.1155 / 2012 / 943635$.

4. Sharan C, Halder SK, Thota C, et al. Vitamin D inhibits proliferation of human uterine leiomyoma cells via catechol-Omethyltransferase. Fertil Steril 2011;95(1):247-253. DOI: 10.1016/ j.fertnstert.2010.07.1041.

5. Halder S, Sharan C, Al-Hendy A. Vitamin D treatment induces dramatic shrinkage of uterine leiomyomas growth in the Eker rat model. Fertil Steril 2010;94(4):S75-S76. https://doi.org/10.1016/ j.fertnstert.2010.07.293

6. Deeb KK, Trump DL, Johnson CS. Vitamin D signalling pathways in cancer: potential for anticancer therapeutics. Nat Rev Cancer 2007;7(9):684-700. DOI: 10.1038/nrc2196.

7. Bläuer M, Rovio PH, Ylikomi T, et al. Vitamin D inhibits myometrial and leiomyoma cell proliferation in vitro. Fertil Steril 2009;91(5):1919-1925. DOI: 10.1016/j.fertnstert.2008.02.136.

8. Halder SK, Goodwin JS, Al-Hendy A. 1,25-Dihydroxyvitamin D3 reduces TGF-beta3-induced fibrosis-related gene expression in human uterine leiomyoma cells. J Clin Endocrinol Metab 2011;96(4):E754-E762. DOI: 10.1210/jc.2010-2131.

9. Ingala P, Mboloko J, Tshiband A, Lepira F, Kayembe P, Lebwaze $B$, et al. Vitamin D Deficiency and Risk of Uterine Leiomyoma among Congolese Women . A Hospital-Based Case-Control Study. American Scientific Research Journal for Engineering, Technology, and Sciences (ASRJETS) (2016) Volume 22, No 1, pp 126-137.

10. Sabry M, Halder SK, Ait Allah ASA, et al. Serum vitamin D3 level inversely correlates with uterine fibroid volume in different ethnic groups: a cross-sectional observational study. Int J Womens Health 2013;5(1):93-100. DOI: 10.2147/IJWH.S38800.

11. Ciavattini A, Carpini GD, Serri M, et al. Hypovitaminosis D and "small burden" uterine fibroids: opportunity for a vitamin $D$ supplementation. Medicine (United States) 2016;95(52):e5698. DOI: 10.1097/MD.0000000000005698. 